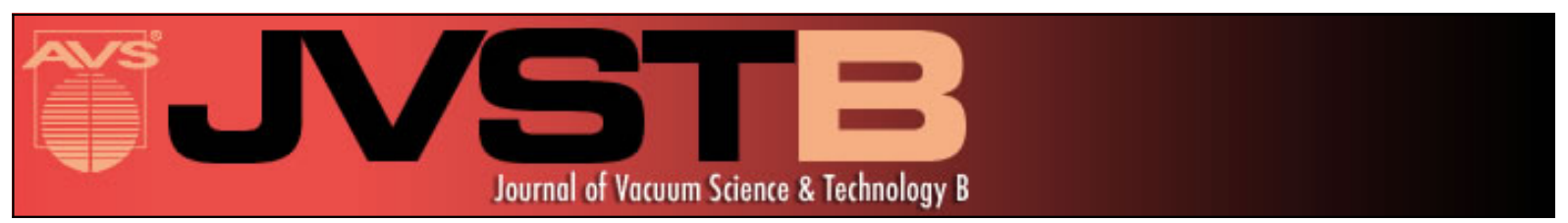

\title{
A low-temperature atomic force/scanning tunneling microscope for ultrahigh vacuum
}

F. J. Giessibl, Ch. Gerber, and G. Binnig

Citation: Journal of Vacuum Science \& Technology B 9, 984 (1991); doi: 10.1116/1.585441

View online: http://dx.doi.org/10.1116/1.585441

View Table of Contents: http://scitation.aip.org/content/avs/journal/jvstb/9/2?ver=pdfcov

Published by the AVS: Science \& Technology of Materials, Interfaces, and Processing

\section{Articles you may be interested in}

Low-Temperature Ultrahigh-Vacuum Atomic Force Microscope

AIP Conf. Proc. 696, 196 (2003); 10.1063/1.1639695

A low-temperature ultrahigh vacuum atomic force microscope for biological applications

Rev. Sci. Instrum. 74, 1022 (2003); 10.1063/1.1532840

A scanning force microscope with atomic resolution in ultrahigh vacuum and at low temperatures

Rev. Sci. Instrum. 69, 221 (1998); 10.1063/1.1148499

A simple low-temperature ultrahigh-vacuum scanning tunneling microscope capable of atomic manipulation

Rev. Sci. Instrum. 67, 2960 (1996); 10.1063/1.1147080

Low-temperature ultrahigh-vacuum scanning tunneling microscope

Rev. Sci. Instrum. 60, 3109 (1989); 10.1063/1.1140586

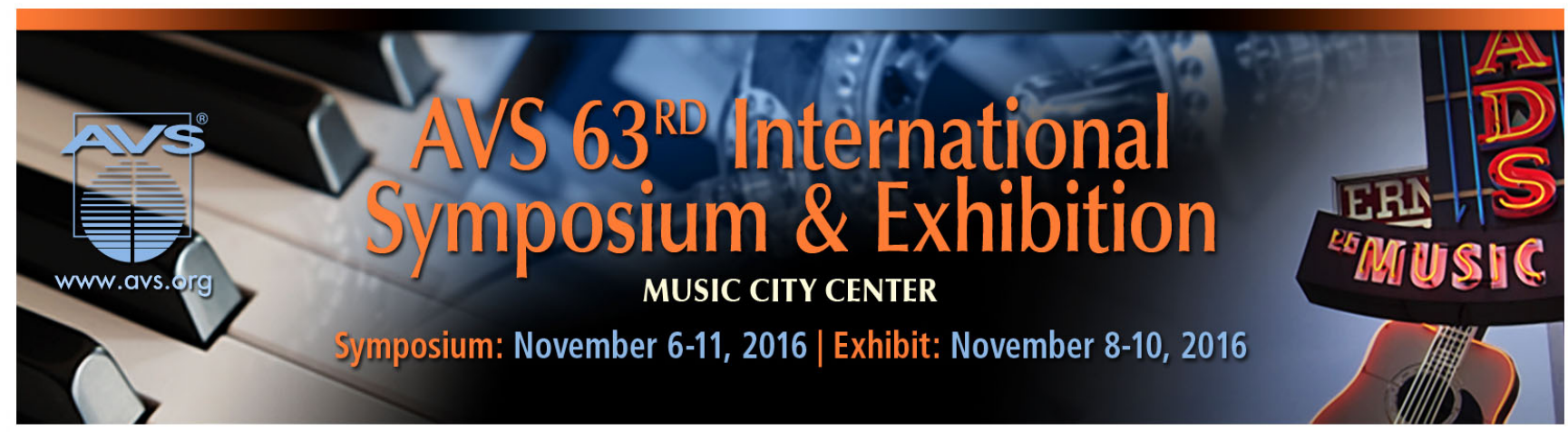




\title{
A low-temperature atomic force/scanning tunneling microscope for ultrahigh vacuum
}

\author{
F. J. Giessibl, Ch. Gerber, ${ }^{\text {) }}$ and G. Binnig \\ IBM Research Division, Physics Group Munich c/o Universität München, Schellingstrasse 4, D-8000 \\ München 40, Germany
}

(Received 30 August 1990; accepted 4 December 1990)

\begin{abstract}
We have built an ultrahigh vacuum atomic force/scanning tunneling microscope that works at $4.2 \mathrm{~K}$. The microscope is incorporated into a very small chamber $(100 \mathrm{ml})$ which can be evacuated and baked to ultrahigh vacuum (UHV) within a few hours by a specially designed valve. The instrument is about $20 \times 20 \times 70 \mathrm{~mm}^{3}$ in size and sturdy enough to operate without vibration isolation. The deflection of a microfabricated cantilever is detected by electron tunneling. Preliminary results show atomic resolution of highly oriented pyrolytic graphite (HOPG) in the scanning tunneling microscope (STM) mode and steps in KBr that range from one to four lattice constants in height at UHV conditions and $4.2 \mathrm{~K}$.
\end{abstract}

\section{INTRODUCTION}

The atomic force microscope (AFM) has been used in air, ${ }^{1}$ water, ${ }^{2}$ ultrahigh vacuum (UHV), ${ }^{3}$ and liquid helium. ${ }^{4}$ Since its invention in $1986^{1}$ there has been interest in operating an AFM in UHV at $4.2 \mathrm{~K}$. UHV opens up the field of surface science on insulators. Low-temperature phenomena like adsorbed gas layers could be studied by a low-temperature AFM (LTAFM).

Owing to the complexity of the experimental problems, a functioning AFM that combines UHV and low-temperature ability has not yet been built as far as we know.

In this paper we describe the assembly of our AFM/STM (scanning tunneling microscope) and vacuum system, and present initial results. We found that at true UHV conditions tunneling is an appropriate method to detect the deflection of the cantilever.

\section{EXPERIMENTAL SETUP}

Figure 1 is a sketch of our UHV system. ${ }^{5}$ The AFM/STM sits in a very small UHV chamber, which can be cooled to 4.2 $\mathrm{K}$ by immersing it into liquid helium. This UHV chamber has to be vented quite often for the following reasons:

(i) Design criteria were not available at the time this LTAFM was planned so it was clear that the instrument would have to go through a few evolution cycles before it would function properly.

(ii) It is not possible to interchange samples, cantilevers or tips in situ in our microscope. Instead, the microscope is built onto a single CF 38 flange supporting all the mechanical and electrical feedthroughs necessary to operate it. The microscope can be taken out of the chamber by loosening six screws. Thus, all parts of the microscope are easily accessible.

We designed the UHV system such that it would allow a short time to pump and bake the AFM/STM chamber after venting. To achieve this, the vented volume was kept small $(100 \mathrm{ml})$ and a special valve was designed that seals two vacuum lines (in two concentric pipes) directly at the AFM/STM chamber. Figure 2 shows a cross section of this valve. The seals consist of gold rings on one side and chromed steel cones on the other. The inner pipe serves as a bypass line. To start the pumping process, the AFM/STM is mounted onto the chamber and the inner valve (bypass valve) is opened. It is pumped by a $1701 / \mathrm{s}$, a $50 \mathrm{l} / \mathrm{s}$ turbo pump and a roughing pump in series. The small chamber is surrounded by heater tape and baked at about $180^{\circ} \mathrm{C}$ for several hours (end pressure approximately $10^{-7} \mathrm{mbar}$ at $180^{\circ} \mathrm{C}, 2 \times 10^{-10}$ mbar at $20^{\circ} \mathrm{C}$ ). Then the inner valve is closed (at $T=180^{\circ} \mathrm{C}$ ) and the outer one opened. The main pumping line remains at UHV during the entire venting and bakeout process. When the valve that connects the main pumping line with the AFM chamber is opened, the pressure at IG 5 (see Fig. 1) rises to $10^{-8} \operatorname{mbar}$ (IG $1: 1 \times 10^{-10}$ mbar) and decreases to approximately $3 \times 10^{-10}$ mbar (IG 1: $3 \times 10^{-11}$ mbar) as the chamber reaches room temperature.

The $z$ distance between lever and sample and the $z$ distance between lever and tunneling tip can be controlled mechanically from the outside while the instrument is in UHV and submersed into the liquid helium dewar (Fig. 3). The CF 38 flange that holds the AFM/STM has two linear mechanical feedthroughs ( via welded metal bellows ${ }^{6}$ ) and ten electrical feedthroughs. ${ }^{7}$ The two bellows are driven by micrometer screws turned by stepper motors.

The AFM itself is made mainly of Invar and uses a new kind of sample approach. The sample is held on a piezoelectric tube scanner which sits on a slide. This slide is pressed on to the ball and axle by a spring-loaded ball bearing [Fig. 4 (a) ]. By turning the axle, the slide rolls on the ball and axle and gently approaches the sample. This feature provides a very smooth approach and avoids the danger of crushing the cantilever between the tunneling tip and sample. This is especially important because in UHV and at low temperatures friction is enhanced and the danger of slip-stick motion increased. Because the sensitivity (displacement per voltage) and thus the full range motion of the piezoelectric tube scanner at $4.2 \mathrm{~K}$ is only about $25 \%$ compared to $300 \mathrm{~K}$ the precision of the mechanical approach has to be even better at liquid helium temperature. The $x$ and $y$ coordinates of the microfabricated cantilever have to be adjusted by hand within $\pm 5 \mu \mathrm{m}$ with respect to the tunneling tip while the instrument is in air at room temperature. Owing to the small size of the instrument and the use of Invar the cantilever and tunneling tip remain aligned as the instrument is cooled to liquid 


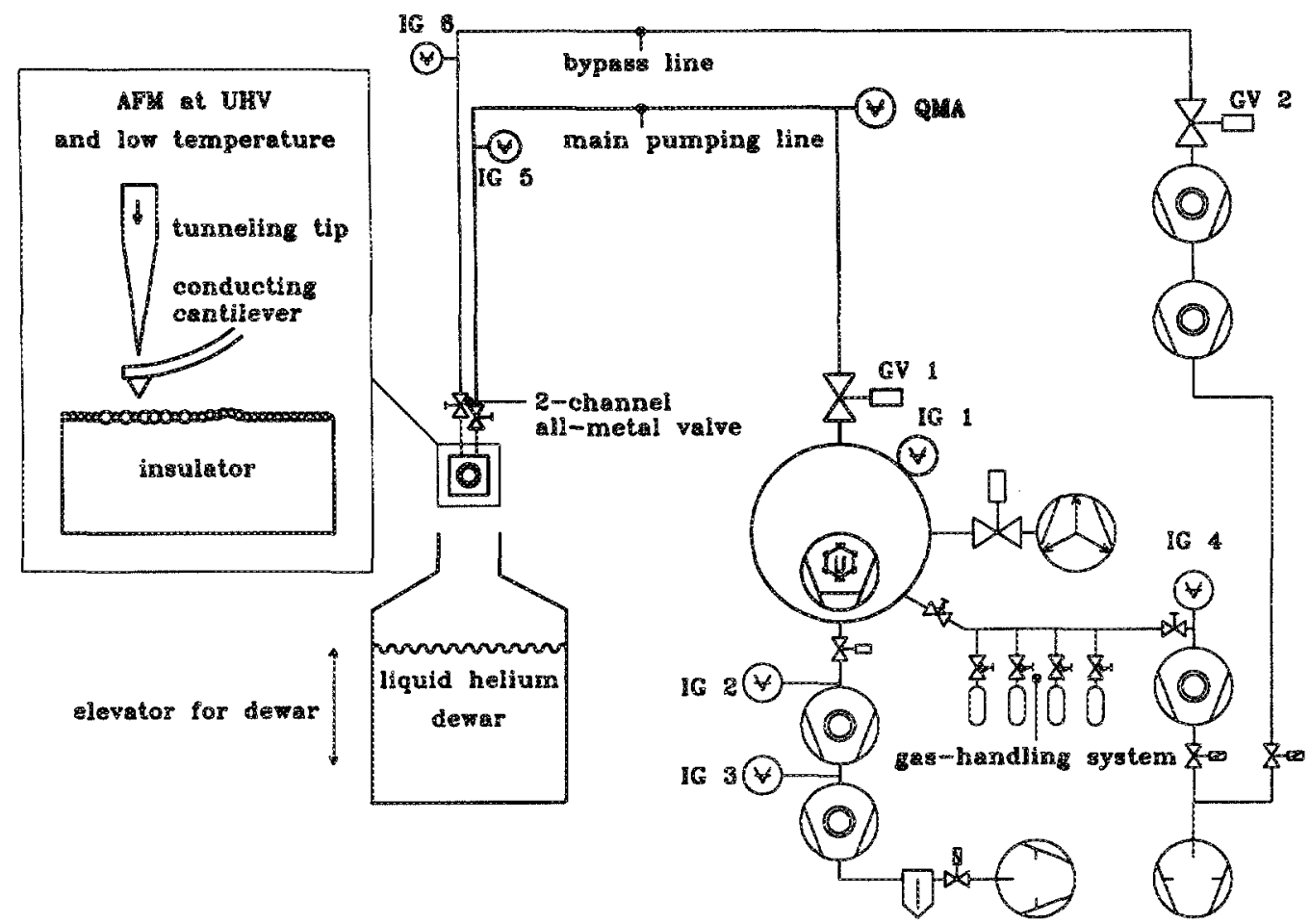

FiG. 1. UHV system showing main vacuum system, bypass line, and gas-handling system. Main pumping line and bypass line can be sealed directly at the chamber to minimize the surface area exposed to air while venting. The liquid helium dewar has a neck width of 80 mm. Bypass and main pumping line are arranged in concentric pipes in order to achieve the optimal conductivity. The gas-handling system is used for dosing small amounts of gas onto a substrate. There are six ion gauge meters (IG 1..6) to monitor the pressure and a quadrupole mass analyzer (QMA) for rest gas analysis, leak checking, and measuring the partial pressures of the gases that are dosed in by the gas-handling system. Although we cannot measure the pressure insice the AFM chamber (it is to small for normal ion gauge heads), the pressure is found by interpolation between IG 5 and IG 6 to be around $7 \times 10^{-10}$ mbar at room temperature. When gate valve no. 2 (GV 2) is closed and both channels of the all-metal valve at the chamber are open, the pressure in IG 5 is $2 \times 10^{-10}$ mbar, while $1 G$ reads $5 \times 10^{-9}$ mbar. At $4.2 \mathrm{~K}$, we expect the pressure to be virtually zero (except for the partial pressure of helium), because we can close the two-channel valve and any residual gases except for helium would freeze onto the walls of the UHV chamber. The main recipient is pumped by an ion getter pump and a titanium sublimation pump. The end pressure in the main recipient is $3 \times 10^{-11}$ mbar. The UHV system was developed in cooperation with and built by VG SPECIA $L$ SYSTEMS, Hastings, England.

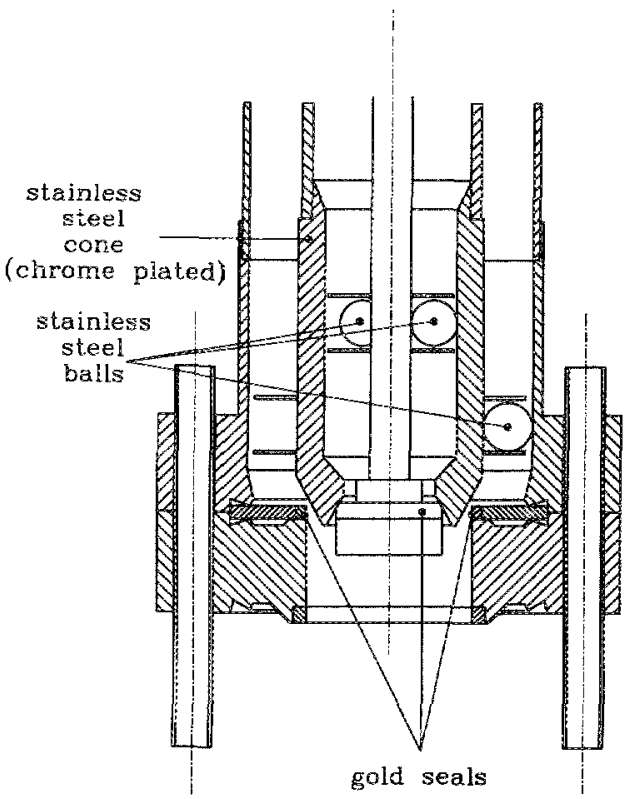

Fig. 2. All-metal UHV valve with two channels to seal the chamber to main and bypass line. The stainless steel balls guide the three main parts of the valve in horizontal direction and allow a vertical movement to open or close the valve. The central part seals the bypass line and consists of a rod with a nut at the lower end. The nut has a gold ring welded to it. The main pumping line is sealed by the stainless steel cone which can be pressed onto a gold ring (the gold ring is welded into the copper seal that seals the two CF 38 flanges against each other)

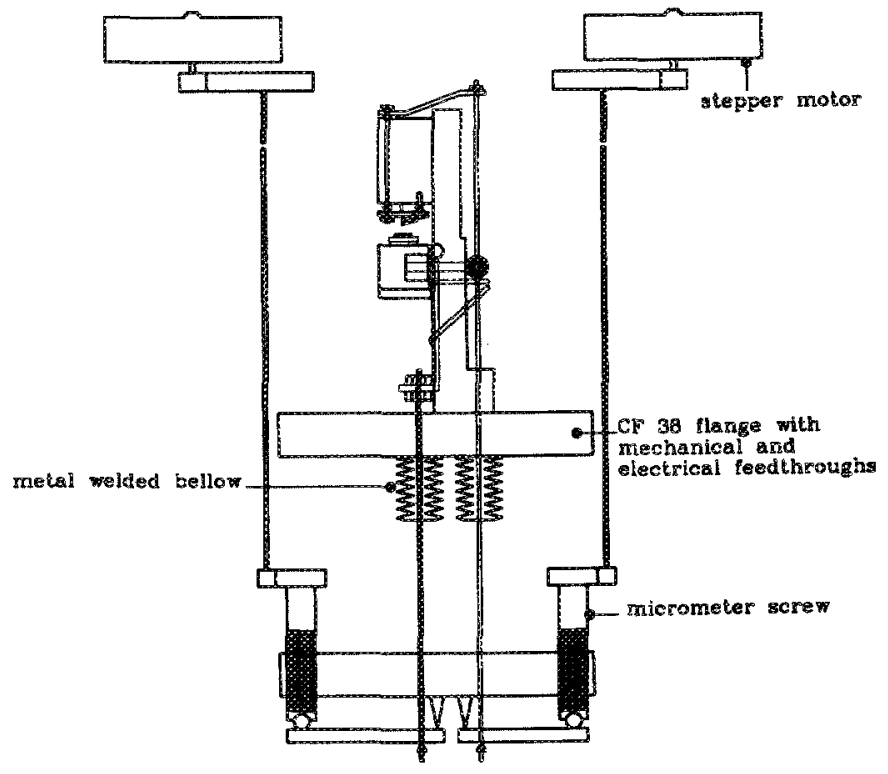

FIG. 3. AFM with mechanical approach mechanism for the $z$ distance between sample, lever and tunneling tip. The electrical feedthroughs are omitted for clarity. The micrometer screws are also immersed into liquid helium and controlled by stepper motors and gears via $0.5-\mathrm{m}$-long shafts (not to seale in this figure). The CF 38 flange that supports the microscope has ten electrical and two mechanical linear feed throughs. ${ }^{7.8}$ 

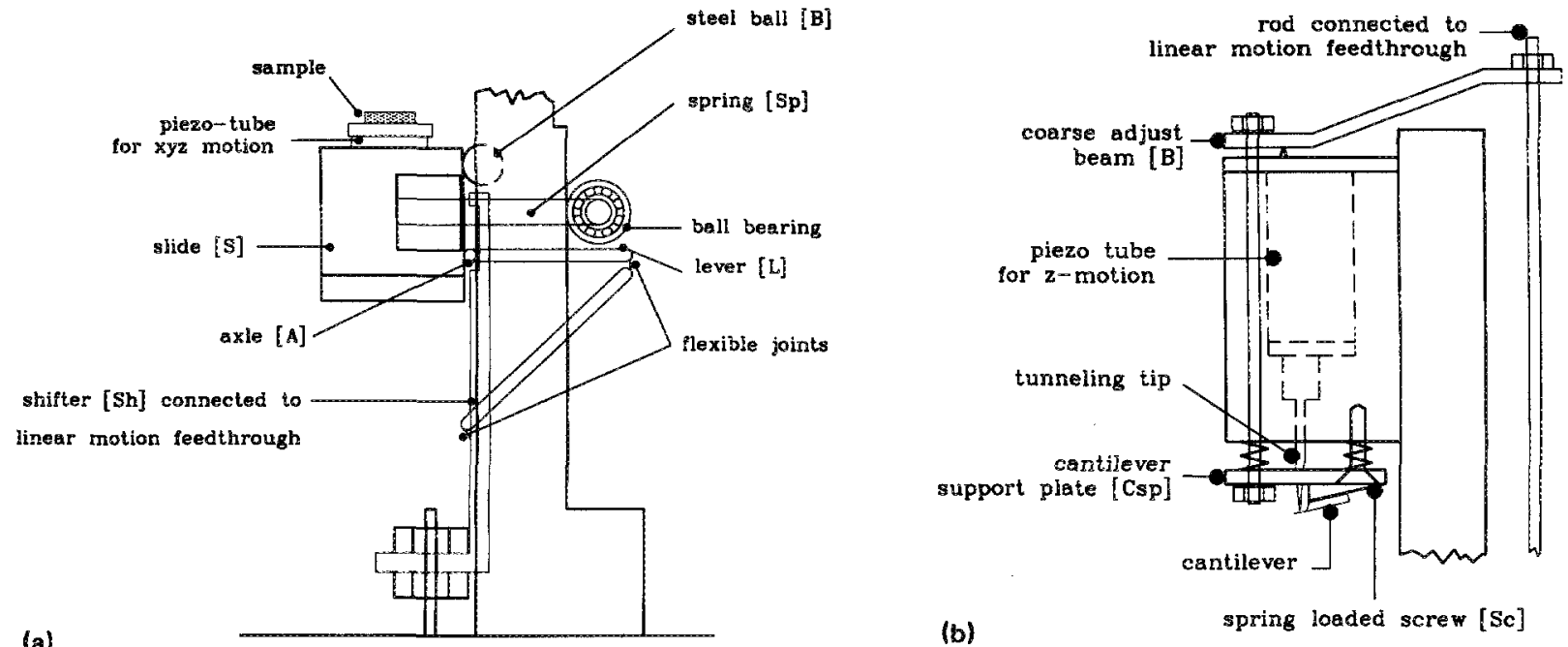

FIG. 4. (a) A pproach mechanism for the $z$ distance between sample and lever. The slide carrying the sample is pressed onto a steel ball (B) and an axle (A) by a spring (Sp) which acts on a ball bearing. The slide is held in position by friction between the slide, the axle (A) and the block that holds the entire instrument. Thus by turning the axle (A) the slide is rolling gently in a vertical direction. The approach is done as follows: the shifter (Sh) is moved upwards until the lower end of its gap (approx. $4 \mathrm{~mm}$ wide) touches the axle (A). Then the entire slide is moved upwards until the sample is about $200 \mu \mathrm{m}$ away from the cantilever. Then the shifter is pulled downwards, setting the axle (A) free and turning the lever (L). By turning the lever, the axle is rotating and thereby further approaching the slide (S) towards the cantilever. Lever and axle are made of $1 \mathrm{~mm}$ steel wire, the length of $(L)$ is $10 \mathrm{~mm}$, so the motion of the shifter is demagnified by a factor of 10. The flexible joints are made by spot welded sheet metal. (b) Approach mechanism for the $z$ distance between lever and tunneling tip. The tunneling tip sits at the end of a piezotube. The microfabricated cantilever is held on its support plate (Csp) and pivots around a springloaded screw ( $\mathrm{Se}$ ). The coarse adjust beam $(\mathbf{B})$ demagnifies the motion of the rod which is connected to one of the linear feedt hroughs. By pulling at the linear feedthrough, the course adjust beam pulls the cantilever support plate against the tunneling tip. The cantilever is plated with chrome and gold; the tunneling tip is made of tungsten.

helium temperature. However, care has to be taken when mounting the tunneling tip. We found that stress relief in the tunneling-tip mount during bakeout can move the tunneling tip up to $50 \mu \mathrm{m}$ sideways. After having been baked once the tunneling gap remained aligned after going through cooling and baking cycles. The distance between the cantilever ( $z$ coordinate) and the tunneling tip is also adjusted in air, and is controlled by a stepper motor while the instrument is in UHV and at liquid helium temperature. The force sensor consists of a housing for a piezotube that holds the tunneling tip and a spring-loaded cantilever support plate. The distance between cantilever and tunneling tip can be adjusted mechanically by leveraging [Fig. 4(b)].

We used PZT 5A as piezoelectric material, ${ }^{8}$ because it has a fairly high Curie temperature (i.e., the temperature at which it becomes unpolarized and therefore loses sensitivity) and thus can be soldered. The tube scanner and the piezoelectric tube that controls the distance between tunneling tip and cantilever were soldered to metal plates on one end with a lead-free solder. The other end was soldered to a slab of metal-coated alumina.

We tested the instrument in the STM mode with highly oriented pyrolytic graphite (HOPG) (Fig. 5). The HOPG was cleaved in air and then baked at $180^{\circ} \mathrm{C}$ for several hours. It took two hours from the time that the liquid helium dewar was lifted (thus submersing the STM chamber in liquid helium) until all the parts of the STM had reached $4.2 \mathrm{~K}$. At that point thermal drift was no longer noticeable.
The micrometer screws can be turned while immersed in liquid helium if they are thoroughly degreased and lubricated with graphite powder.

Next we tested the instrument in the AFM mode using $\mathrm{KBr}$ (an alkali halide like $\mathrm{NaCl}$ and $\mathrm{LiF}$ ) as a sample. The $\mathrm{KBr}$ crystal was cleaved in air at its natural (100) cleavage plane, mounted onto the AFM and baked together with the microscope at $180^{\circ} \mathrm{C}$ for four hours. The cleavage plane looked mirror-like for sections of several $\mathrm{mm}^{2}$. The approach between tunneling tip and cantilever was done before cooling the instrument to $4.2 \mathrm{~K}$ and the width of the tunneling gap was controlled by one of the stepper motors during the cooling cycle. After two hours, we started to approach the sample towards the cantilever. Steps of a height ranging from one to several lattice constants could be resolved (Fig. 6). However, we have not yet succeeded in imaging the unit cell.

At room temperature, atomic resolution on $\mathrm{NaCl}$ in UHV has recently been observed by Meyer and Amer with an AFM that uses an optical detection method." Meyer et al. ${ }^{10,11}$ were able to image $\mathrm{LiF}$ at atomic resolution in ambient pressure and room temperature with an AFM that uses electron tunneling to measure the deflection of the cantilever.

\section{CONCLUSION}

For the first time, as far as we know, an AFM was successfully operated at UHV and $4.2 \mathrm{~K}$. So far, we have only 


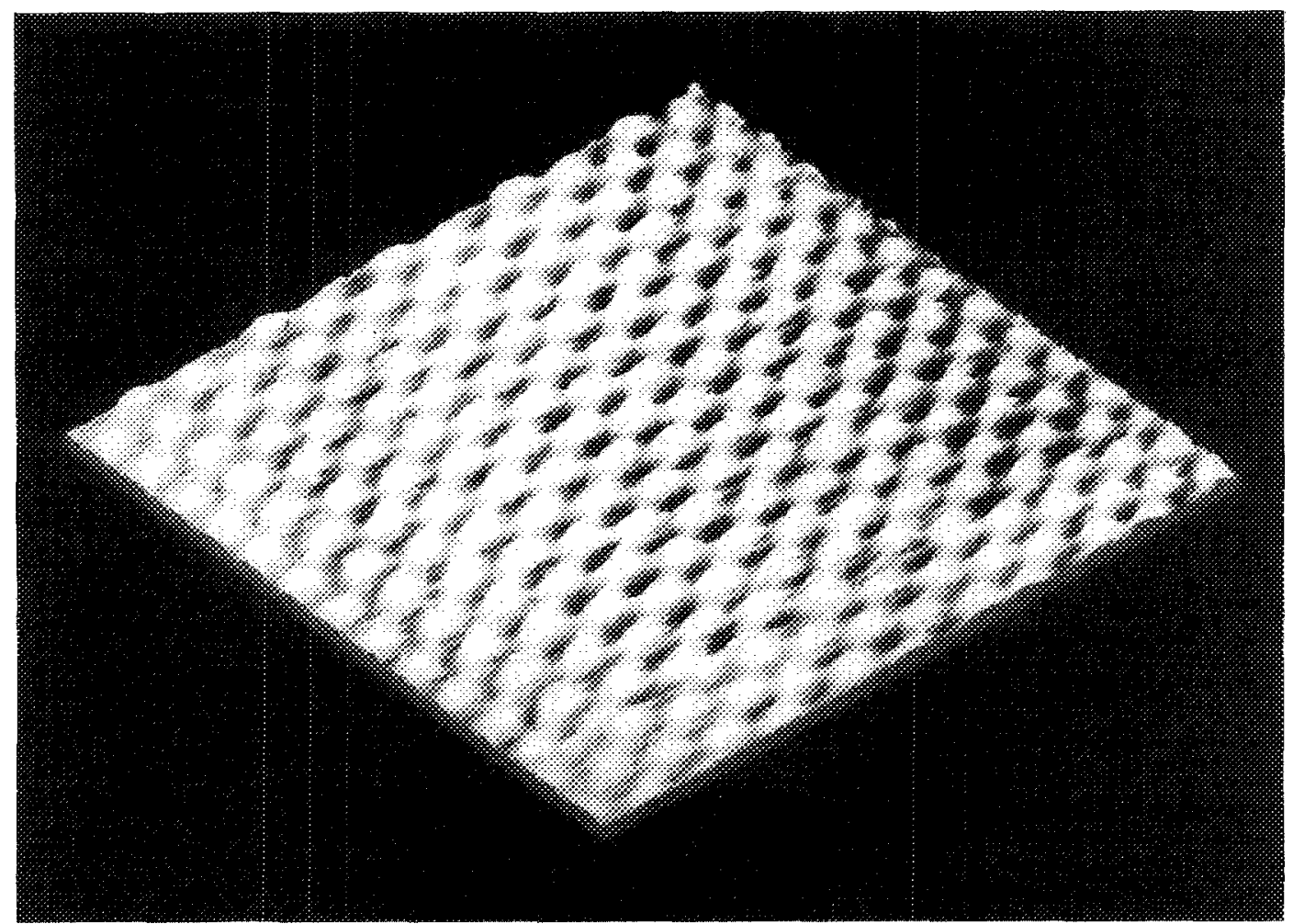

Fig. 5. HOPG at $4.2 \mathrm{~K}$ in UHV (raw data) in constant-current inaging ( $1 \mathrm{nA}$ at $600 \mathrm{mV}$ ). Scan area $3.5 \times 3.2 \mathrm{~nm}^{2}$.

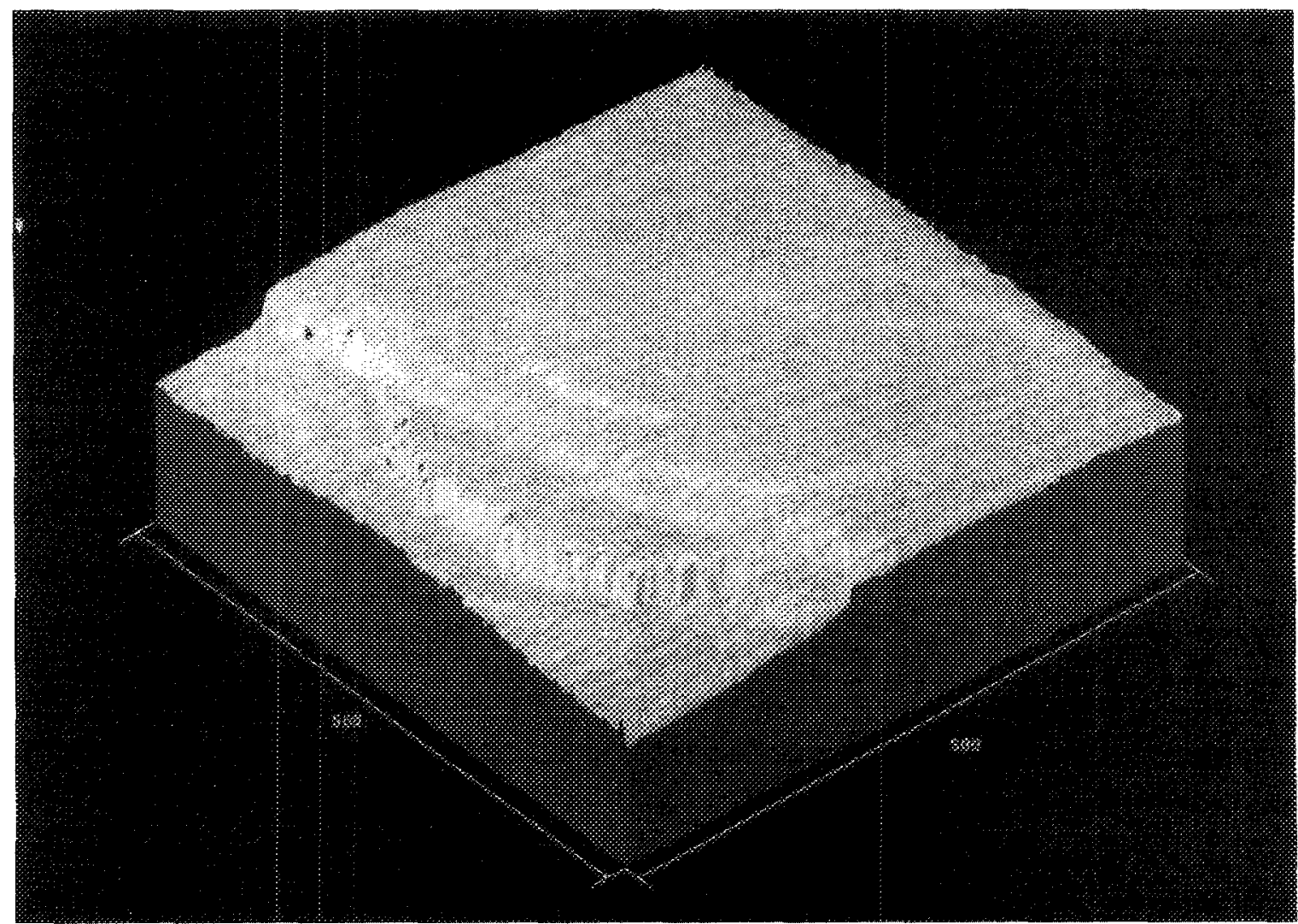

FrG. 6. KBr at $4.2 \mathrm{~K}$ in UIV, raw data-constant force mode-total force between sample and cantilever approximately $4 \times 10{ }^{4} \mathrm{~N}$ (attractive). Scan area $58 \times 58 \mathrm{ntn}^{2}$. 
imaged steps on $\mathrm{KBr}$. We have achieved atomic resolution in the STM mode on HOPG but not on $\mathrm{KBr}$ in the AFM mode. We believe the reason for this is that the $z$ resolution of our instrument in the AFM mode is not yet sufficient for detecting the rather small corrugation of $\operatorname{KBr}(0.062 \mathrm{~nm})$. The cantilever support plate [see Fig. 4(b)] with its mechanical adjusting mechanism is affected to a greater extent by mechanical vibrations than are the other parts of the microscope. Therefore the tunneling current is modulated more strongly by mechanical vibrations in the AFM mode than in the STM mode. The source of this noise are mechanical vibrations of the crate that carries the UHV system. The dom:nant frequency components of the mechanical vibrations in the AFM/STM chamber are integer multiples (200, 300, $400 \mathrm{~Hz}$ ) of the line frequency $(50 \mathrm{~Hz})$. They are generated by fans, transformers, etc., and coupled to the vacuum chamber by sound waves through air and via the foor of the laboratory.

\section{OUTLOOK}

We believe that the possibility of using AFM in UHV at $4.2 \mathrm{~K}$ opens up a new field of research on insulators and even on individual molecules adsorbed on a substrate. Both lateral diffusion across a substrate and desorption of a molecule are strongly reduced at low temperature [the rates vary at approximately $\left.\exp \left(-E_{\text {bind }} / k T\right)\right]$. We expect to be able to image samples which are now too soft to be investigated by an AFM operating at room temperature. The absence of drift allows the scanning speed to be set at any appropriate value to produce the best image quality. Furthermore, it is much easier to apply image averaging techniques if consecutive images are not displaced by thermal drift. Currently, mechanical vibrations are the dominant source of noise in our microscope. We are therefore working on a vibration insulation stage directly at the microscope and are endeavoring to diminish the mechanical noise amplitude that is coupled into our vacuum system.

\section{ACKNOWLEDGMENTS}

We would like to thank James Chapman from Standard Bellows ${ }^{6}$ for making excellent welded metal bellows for us in a very short time, and A. Ebenböck and his team for machining most of the parts of the STM and AFM.

a) IBM Research Division, Zurich Research Laboratory, Säumerstr. 4, CH8803 Rüschlikon, Switzerland.

${ }^{\prime}$ G. Binnig, C. F. Quate, and Ch. Gerber, Phys. Rev. Lett. 56, 939 (1986).

${ }^{2}$ S. Gould, O. Marti, L. Hellemans, C. E. Brecker, P. K. Hansma, N. L. Keder, M. M. Eddy, and G. D. Stucky, Nature 332, 332, (1988).

G. Meyer and N. M. Amer, Appl. Phys. Lett. 53, 1045 (1988).

${ }^{4}$ M. D. Kirk, T. R. Albrecht, and C. F. Quate, Rev. Sci. Instrum. 59, 833 (1988).

"VG Special Systems Ltd., Hastings, England.

${ }^{6}$ Standard Welded Bellows, Windsor Locks, CN.

${ }^{7}$ Ceramaseal, New Lebanon, NY.

${ }^{8}$ Stavely Sensors, East Hartford, CN.

"G. Meyer and N. M. Amer, Appl. Phys. Lett. 56, 2100 (1990).

${ }^{10}$ E. Meyer, H. Heinzelmann, H. Rudin, and H.-J. Güntherodt, Z. Phys. B 79, 3 (1990).

${ }^{1 '}$ E. Meyer, H. Heinzeimann, D. Brodbeck, G. Overney, R. Overney, I. Howald, H. Hug, T. Jung, H.-R. Hidber, and Ih.J. Güntherodt, J. Vac. Sci. Technol. B 9, 1329 (1991). 